FedUni ResearchOnline

http://researchonline.federation.edu.au

This is the peer-reviewed version of the following article:

Hassan, M., Karmakar, G., Kamruzzaman, J., Srinivasan, B. (2015) A comprehensive spectrum trading scheme based on market competition, reputation and buyer specific requirements. Computer Networks, 84, 17-31.

Which has been published in final form at:

http://doi.org/10.1016/j.comnet.2015.04.004

Copyright $\odot 2015$ Elsevier Ltd. This manuscript version is made available under the CC-

BY-NC-ND 4.0 license http://creativecommons.org/licenses/by-nc-nd/4.0/ 


\title{
A Comprehensive Spectrum Trading Scheme Based on Market Competition, Reputation and Buyer Specific Requirements
}

\author{
Md. Rakib Hassan ${ }^{\mathrm{a}, \mathrm{b}, *}$, Gour Karmakar ${ }^{\mathrm{b}}$, Joarder Kamruzzaman ${ }^{\mathrm{a}, \mathrm{b}}$, Bala \\ Srinivasan $^{\mathrm{a}}$ \\ ${ }^{a}$ Faculty of Information Technology, Monash University, Victoria, Australia \\ ${ }^{b}$ Faculty of Science, Federation University, Victoria, Australia
}

\begin{abstract}
In the exclusive use model of spectrum trading, cognitive radio devices or secondary users can buy spectrum resources from licensed users or primary users for a short or long period. Considering such spectrum access, a trading model is introduced where a buyer can select a set of candidate sellers based on their reputation and their offer in fulfilling its requirements, namely, offered signal quality, contract duration, coverage and bandwidth. Similarly, a seller assesses a buyer as a potential trading partner considering its reliability, which the seller can derive from the buyer's reputation and financial profile. In our scheme, seller reputation or buyer reliability can be either obtained from a reputation brokerage service, if exists, or calculated using our model. Since in the competitive market, the price of a seller depends on that of other sellers, game theory is used to model the competition among multiple sellers. An optimization technique is used by a buyer to select the best seller(s) and optimize purchase to maximize its utility, which may result in buying from multiple sellers, of certain amount of bandwidth from each, depending on price, fulfilling requirements and budget constraints. Stability of the model is analyzed and performance evaluation
\end{abstract}

\footnotetext{
* Corresponding author

Email addresses: rakibbau@gmail.com (Md. Rakib Hassan), gour.karmakar@federation.edu.au (Gour Karmakar), joarder.kamruzzaman@federation.edu.au (Joarder Kamruzzaman), srini@monash.edu (Bala Srinivasan)
}

Preprint submitted to Computer Networks

October 20, 2014 
shows that it benefits both sellers and buyers in terms of profit and throughput, respectively.

Keywords: Cognitive radio networks, spectrum trading, reputation, recommendations, game theory.

2010 MSC: 00-01, 99-00

\section{Introduction}

Cognitive radio (CR) enabled devices termed as secondary users (SUs) can solve the problem of spectrum scarcity [1, 2] in the unlicensed band by sharing the under-utilized licensed band with the licensed or primary users (PUs). This

5 spectrum sharing has been categorized in three models - commons, shared-use and exclusive-use models [3. Commons model is applied in unlicensed bands (e.g. ISM bands) where spectrum can be accessed by everyone and hence, becoming congested due to the increased usage and number of wireless devices. In shared-use model, SUs sense the licensed spectrum to exploit it opportunistically i.e., they use the licensed spectrum when it is not used by PUs. Although SUs can access the spectrum without any cost in this model, it has several limitations 4, 5. For example, continuous sensing and careful deployment of SUs are required, communication overhead increases and finding and switching to a free channel may become difficult because spectrum holes may not be always 15 available.

On the other hand, exclusive-use model has several advantages and do not suffer from the above mentioned limitations. In the exclusive-use model, SUs have exclusive rights to access the spectrum by buying channels from primary service providers, hereafter termed as primary services, for a shorter or longer ${ }_{20}$ period of time without any need for spectrum sensing. Besides, SUs can buy the spectrum at a lower price and the primary services can make some profit by selling their unused resources to the SUs, benefitting both the primary and the secondary services. This type of spectrum access model between the primary and the secondary services has been adopted in different contexts exploiting 
different solution techniques [6-14].

In [15], we introduced a spectrum trading model using exclusive-use model where a buyer chooses a seller based on its reputation, service contract duration, signal quality and bandwidth of the offered spectrum. But we did not consider the reputation of buyers as seen from sellers' perspective for trading purpose.

30 The consideration of a buyer's reputation is also of paramount importance for a successful leasing based trading. Therefore, in this paper, we have incorporated several aspects to elevate the trading of spectrum to a more realistic level. First, we have introduced a buyer's reputation and credit score which can be used as criteria by a seller to assess the reliability of a buyer. This is essential 35 in buyer selection when the payments of the contract are in installments or in post-paid basis. Similar to [15], a buyer's reputation is calculated based on a seller's own experience and recommendations from other sources. Experience is calculated based on payment completion, timely payment and percentage of contract completion of a spectrum lease. Trustworthiness of recommenders is 40 also considered important to reduce the impact of false recommendations.

In the traditional credit rating system, there exist many agencies (e.g., Standard \& Poor's, Moody's and Fitch Ratings) who assess the creditworthiness of a company or a government, while the others such as credit bureau and consumer reporting agency collect and evaluate credit information of individual

45 consumers for applications such as bank loan, leasing, employment, etc. [16]. Currently, there exists no reputation/reliability brokering service for spectrum trading. However, with increased business opportunities of spectrum trading, such brokering service is expected to become available in the future. Therefore, in our model, we keep provisions for a seller to obtain the reliability score of a 50 buyer from either a broker or by the seller's own analysis.

Second, we consider competition among sellers and model this competition using non-cooperative game theory to determine the Nash equilibrium trading price. In [15], we assumed inherent competition among sellers, and therefore, used market equilibrium to determine the supply and demand of the spectrum 55 bandwidth. In the case of multiple sellers and buyers, market equilibrium cal- 
culates the combined supply of sellers as a single supply curve and that of the buyers as a single demand curve. This was sufficient in [15] because sellers were assumed to be unaware of the spectrum price of other sellers. But if sellers are aware of the prices of other sellers and they compete with each other in maximizing their own profits, and in such case non-cooperative game theory is the best candidate to model this price competition. Therefore, in this paper, we use non-cooperative game theory to determine the pricing solution. Third, we consider two types of buyers in the market: (i) price sensitive and (ii) price insensitive. Price sensitive buyers are those who buy spectrum from sellers and maximize its utility or satisfaction within its budget constraint. In contrast, price insensitive buyers are focused on buying their required bandwidth rather than considering price as a constraint.

Thus, in the proposed model, a buyer and a seller will be aware of each other to ensure successful trades. The major contributions of this paper can be summarized as:

- The selection of buyers is based on their reliability, which can be either obtained from a broker or calculated by a seller considering the buyer's financial profile, seller's own past trading experience and recommendations from other sources about that buyer. Experience is numerically quantified utilizing payment completion, timely payment and percentage of completion of contract duration, while the creditworthiness of a buyer is theoretically modeled using a generalized credit scoring model using logistic regression.

- Introduction of competition among sellers and its theoretical model with a non-cooperative game theory.

- Modelling of two types of buyers, namely, price sensitive and insensitive buyers, and their analytical solutions for required amount of bandwidth purchase maximizing utility functions.

- Analysis of the proposed model against existing popular and recent models 
[15, 17] in terms of throughput and economic benefits.

The rest of the paper is organized as follows: related works of spectrum trading using game theory are described in Section 2, Section 3 describes the system model with the assumptions considered in this paper. Buyer selection process is presented in Section 4. Section 5 represents competition among sellers, while Section 6 describes the analytical solution used by a buyer in buying the spectrum. Performance evaluation is presented in Section 7 while Section 8 concludes the paper.

\section{Related Works}

Spectrum trading had been applied to solve the pricing issue in network

95 auctioning, classical optimization, game theory and microeconomic approach. In auctioning approach, trading is time-dependent where the bidding decision is taken at a certain interval or at a fixed time. Optimization can be used in spectrum trading to maximize or minimize an objective function of a seller or a buyer under some constraints. This objective function can express a trading goal (e.g., maximum profit, minimum cost) in mathematical terms. Game theory is used when a system exists with multiple entities with different objectives and an equilibrium solution is desired rather than satisfying a single objective with a global optimum solution.

105 A spectrum market is considered in [7, where the same seller is evaluated differently to various buyers based on their applications and locations. Stochastic learning algorithm is used to find the optimal price in the presence of limited information (e.g., buyer's utility function and prices of other sellers) in order to maximize the profit of the sellers. But the seller's utility function for selecting buyers is ignored in this work. As a result, sellers are unaware of the nature of demand from the buyers.

In the approach described in 18, PUs lease spectrum to SUs in exchange for cooperation in the form of distributed space-time coding, instead of using any 
pricing model for remuneration. Game theory is used to model the competition 15 This model requires that the SUs will relay the data of the primary transmitters to the primary receivers. However, it cannot be guaranteed that the SUs will cooperate in relaying the data of the PUs. In [11], spectrum sharing among multiple SUs and a single PU is considered. Non-cooperative game theory is used to model the competition for bandwidth among the SUs. PU or seller then calculates the price based on the demand from the SUs or buyers. Here, neither any competition nor cooperation is considered among the PUs. A twotier market was proposed in [19] for spectrum trading. In Tier-1, spectrum is traded from PUs to SUs and Nash bargaining game is used to achieve the fairness between the aggregated utility of all SUs and the utility of a PU. In Tier2 market, spectrum is redistributed among SUs to meet their traffic demands. Random matching and bilateral bargaining is used in this tier to obtain solution. Only a single PU has been considered in this work.

In the approach introduced by Zhu et al. 20, competitive secondary service providers lease spectrum from a spectrum broker. SUs select the service based on an evolutionary game and a differential game is used to model the competition among the secondary providers. Here, SUs start by selecting the providers randomly, and then the strategy with a higher payoff than the average are replicated by other SUs. Replication is achieved by switching to different providers frequently to reach an equilibrium solution, which is time consuming and too frequent switching makes the scheme unfeasible in real market.

In 21, multiple PUs sell spectrum to multiple SUs. SUs evolve over time to buy spectrum resources which maximizes their payoff in terms of transmission rate and price using the evolutionary game theory. Deterministic and stochastic models of evolutionary game are used to model this evolution of SUs. Spectrum size and price are determined by the PUs and non-cooperative game theory is used to model the competition among the PUs. All SUs' net utility are assumed to be the same in a group. It does not consider that each SU's requirements can vary from the others within a group. The authors assumed that all SUs 

spectrum size with the same price.

None of the existing works on spectrum trading including those that used game theory considered reputation of sellers and buyers and their effects on spectrum pricing. They also did not consider trustworthiness of recommenders. Therefore, these works cannot portray the price dynamics of a real market.

\section{System Model}

A network consisting of $N_{y}$ primary services (e.g., spectrum sellers) and $N_{x}$ secondary services (e.g., spectrum buyers) are considered in the proposed model as shown in Fig. 1. Each of the primary and secondary service serves ${ }_{155} n_{y}$ and $n_{x}$ number of primary (PUs) and secondary users (SUs), respectively. These primary services (e.g., TV and radio stations, telecommunication service providers) who own some spectrum resources may sell unutilized portion of their spectrum to secondary services (e.g., secondary base stations, ad hoc networks, enterprise networks) who do not possess any spectrum license. Thus the primary services can earn extra revenue and the secondary services can obtain guaranteed spectrum access with desired spectrum characteristics and requirements.

Buyers (i.e., secondary services) who wish to buy spectrum resources will request price per unit bandwidth and other information of trading offer to the sellers (i.e., primary services). Buyers will build or update their reputation database about the sellers from the recommendations of the other buyers and their own experiences. Sellers will also calculate the reliability of buyers based on their credit scoring and reputation. A seller will compete with other sellers in determining spectrum price based on the demands from buyers. Buyers will then buy spectrum resources from the sellers that maximize their utility functions 170 and satisfy their requirements. 


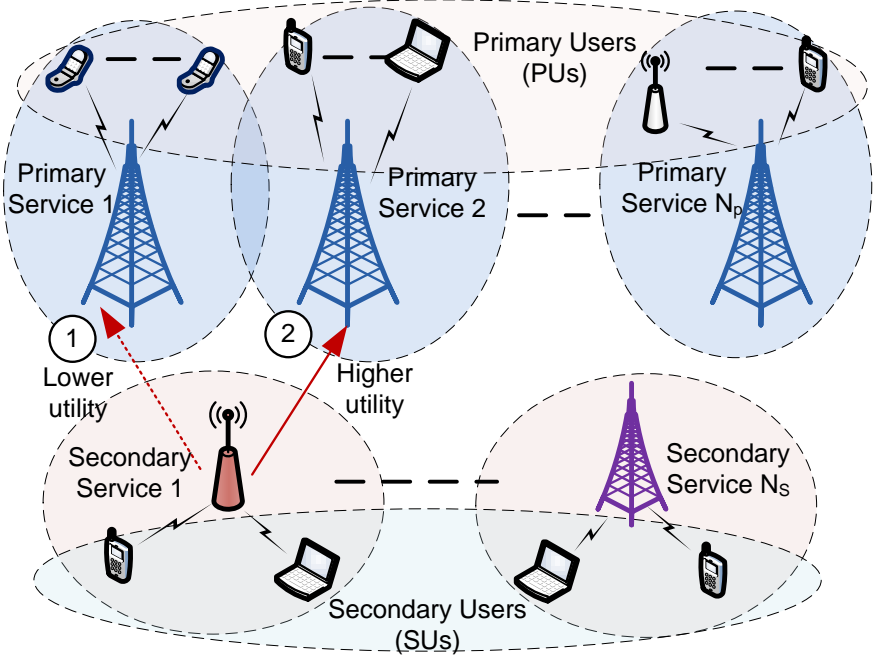

Figure 1: Network model for the spectrum trading between the primary and secondary service providers.

\section{Buyer Selection Process}

Payment for the spectrum resources can be made in two ways: (i) prepaid and (ii) postpaid or in installments (e.g., renting of satellite TV channels). In prepaid transactions, buyers have to clear the payments before using the spectrum resources. However, buyers can pay the fees later in post-paid transactions. Thus in post-paid payment, sellers should check the eligibility of a buyer considering its past payment history and other related characteristics. This eligibility checking is very common financial dealings (e.g., bank loans) where it is termed as 'credit scoring'. It is a method for classifying credit applicants into 'good' or 'bad' risk classes 22. Although the credit assessment for bank loans or other similar loans are different from spectrum trading, the same idea of risk assessment to provide lease to a spectrum buyer can be integrated to improve financial security from a seller's point of view. Furthermore, if there are high demands for limited spectrum resources in the market, sellers would have the opportunity to select a few from many buyers based on the credit scoring criteria along with other factors. Besides this credit scoring, the reputation of buyers is 
also considered in the selection process by a seller to provide priority to highly reliable buyers.

Such reliability score can be obtained in two different ways. One way is through a broker entity. As indicated in Section 1. brokers are the accepted, trusted and neutral entities in various business domains whom the trading partners can rely on for providing scores and can make those publicly accessible or provide on request. A seller $y$ will obtain reliability score of buyers (e.g. buyer $x)$, termed as $\aleph_{x y}$ from the broker. In absence of any such broker in a business domain it is possible for a trading entity to calculate the reliability score of its partner in a transaction using its own past trading experience or collecting information from other sources in a collaborative manner. To the best of our knowledge, no reliability broker on spectrum trading currently exists in practice. In our proposed model, we have kept the provision of utilizing reliability brokerage service if such broker comes into practice in future. In absence of brokerage service currently in spectrum trading, we develop the following model to calculate reliability using the latter approach. Even in the presence of brokerage, such model will be extremely useful in cases where the reliability score of a particular entity is not currently available to broker or an entity prefers its own assessment to that of a broker.

We define the reliability of a buyer as a combination of its reputation and credit score. The reliability of a buyer would be high if both its reputation and credit score are high. This implies the need to use the product of reputation and credit score to measure the reliability. In a study 23, the authors have shown that reputation of a business has a linear relationship with reliability or other risk factors. Such linear relationship between reputation, reliability and other factors are also supported by other studies such as 24 . In our paper, the credit score and reputation covers two aspects by which a buyer can be assessed by a seller and are detailed in Sections 4.1 and 4.2 . Considering these two aspects, the reliability of a buyer is defined as follows:

$$
\aleph_{x y}=\pi_{x y} \varrho_{x y},
$$


where, $\aleph_{x y}, \pi_{x y}$ and $\varrho_{x y}$ are the reliability score, credit score and reputation of buyer $x$ towards seller $y$, respectively. Buyer $x$ is selected by seller $y$ based on a selection score which depends on the seller's business policy.

\subsection{Credit Scoring of Buyers}

There are different methods for credit scoring. Hand and Henley in [22] compared different classification methods (e.g., discriminant analysis, linear regression, logistic regression, genetic algorithms, neural networks) in consumer credit scoring and found that all methods perform almost similar. The performance (e.g., classification accuracy and speed) depends on the model's data one of the most popular statistical tools for credit scoring. Unlike other tools (e.g. discriminant analysis or ordinary linear regression), it can fit various kinds of distribution functions (e.g. Poisson, binomial and normal distributions) and also performs well in fraud detection [25. Logistic regression has been extensively used in credit scoring models [26 31] and is well suited to determine credit scoring of a spectrum buyer.

In the case of post-paid contract, buyers submit their information to a seller and apply for a contract. The seller then assesses the risk involving this buyer. In our approach, a seller employs a generalized credit scoring model using logistic regression [32, 33]:

$$
\pi_{x y}=\frac{e^{B_{0}+\sum_{i=1}^{n} B_{i} X_{i}}}{1+e^{B_{0}+\sum_{i=1}^{n} B_{i} X_{i}}},
$$

where, $\pi_{x y}$ represents the probability which approximates the credit score of buyer $x$ for seller $y ; B_{i}(0 \leq i \leq n)$ are the coefficients of the logistic regression model and $X_{i}$ are the interval scale variables (e.g. revenue and loan).

The required information of a buyer for credit score depends on the nature or the objective of a business. For example, revenue, liability or loan and fixed asset can be considered to determine the credit score of a buyer. In that case, if a buyer has a higher revenue, asset and lower loan, a seller will have a lower probability of having an unsuccessful contract with this buyer. 


\subsection{Reputation of Buyers}

In [34, Liu and Issarny defined reputation as "Reputation of an agent is a perception regarding its behavior norms, which is held by other agents, based on experiences and observation (i.e., indirect observation through peers' recommendations) of its past actions." They mentioned that reputation can be assessed from the following two sources: i) own interaction experience of an assessee with the assessed entity, and ii) recommendations from other peers or users who have interacted with the assessed entity. Therefore, reputation is a perception of the trustworthiness of an entity based on one's own experiences and others' recommendations 35. Besides, reputation is dynamic in nature and can evolve with time [36 38.

In economics, reputation is also defined as the expectation of quality of a product or service [39]. Reputation partially helps in building the loyalty of customers [40, 41]. Thus, reputation can enhance the profitability of an organization because higher customer loyalty generates increased economic returns [42. Landon et al. 39] also found that reputation can determine the price of a product.

The reputation of a buyer to a seller is required if the service or product is traded through a leasing contract. For other situations, such as prepaid service contracts, buyer's reputation is not significant. There are many postpaid services available across the world. For online sales based on leasing, assessing the reputation of a buyer is very crucial to reduce the risk of losing payments where there is no guarantee from a bank. Moreover, it is also important for a trade where a guarantee is achieved from the bank based on conditions, such as regular payments required in a fixed installment.

A seller can calculate the reputation of a buyer based on its own experience and from recommendations from other sources (details on a variety of recommenders is discussed in Section 4.2.1). Therefore, we define buyer $x$ 's reputation 
to seller $y$ from [15] as:

$$
\varrho_{x y}=\frac{\iota_{x y} \zeta_{x y}}{\iota_{x y}+\sum_{j=1}^{R_{x}} \iota_{x j} \phi_{x j}}+\frac{\sum_{j=1}^{R_{x}} \iota_{x j} \phi_{x j} \zeta_{x j}}{\iota_{x y}+\sum_{j=1}^{R_{x}} \iota_{x j} \phi_{x j}},
$$

where, $\iota_{x y}$ is the confidence level of seller $y$ on buyer $x$ 's consistency in past experiences; $\iota_{x j}$ is the confidence level provided by recommender $j$ towards $x$; $\zeta_{x y}$ is the seller $y$ 's own trading experience with $x$ and $\zeta_{x j}$ is the recommendation from a recommender $j ; R_{x}$ is the number of recommenders for $x$ whose trustworthiness to seller $y$ is greater than some predefined value and $\phi_{x j}$ is the trustworthiness of recommender $j$ for buyer $x$. A predefined value is used to filter out recommenders who have lower trustworthiness to the seller. Therefore, 280 the first term of (3) implies a seller's own experience and confidence level about a buyer, and the second term is the collection of recommenders' confidence level and experiences about the buyer.

To measure the confidence level $\iota_{x y}$ about a buyer in trading with a seller, we have used the standard deviation of past experiences. If a buyer gave different experiences at different times, its standard deviation would be high, implying more risk to the seller. Therefore, the confidence level of that seller towards this buyer should be lower. If $T_{x y}^{e}$ is the total number of trading experiences of seller $y$ with buyer $x, \iota_{x y}$ can be calculated as:

$$
\iota_{x y}=\frac{1}{1+\sqrt{\frac{1}{T_{x y}^{e}} \sum_{t=1}^{T_{x y}^{e}}\left(\zeta_{x y}(t)-\bar{\zeta}_{x y}\right)^{2}}} .
$$

If the seller $y$ lacks any previous experience with the buyer $x, \iota_{x y}$ is set to 0

and the seller will depend only on the recommendations from other sources. If the seller itself as well as the recommenders have no experience with that buyer, $\varrho_{x y}$ will be set to 0.5 . In a similar way, the confidence level of a recommender $j, \iota_{x j}$ can be calculated.

Few questions may arise regarding the reputation system of buyers. First, why is reputation system for buyers required? Second, why will the primary users bear the risk of default as there are other third parties (e.g., PayPal) 
who typically evaluate payer's credit-worthiness and assume the corresponding credit risks and management? Third, in which cases may sellers share selective information of their buyers that serves the interest of the seller-community as a whole?

There are several compelling reasons for the inclusion of buyers' reputation in our system model which are discussed below. It is true that there exist third parties such as PayPal who typically assess credit-worthiness of buyers and bear the credit risks. But it is not applicable in all cases. PayPal and most popular third parties provide protection when the items are tangible and shipped to physical addresses. According to PayPal, an item is tangible when it can be held and shipped. Otherwise, it is intangible (e.g., service, eBooks, mp3, etc.) [43]. In this definition, spectrum resources belong to intangible category. Therefore, the spectrum sellers will not be protected from fraudulent buyers if they rely only on these third parties. In these circumstances, the sellers require to manage and prevent the credit risks by themselves.

Besides, if a seller has protection from third parties, it has no need to share buyer's information to other sellers. But when a seller sells intangible items, it is for its own interest to share the updated list of its blacklisted buyers to other sellers so that every seller can protect itself from blacklisted buyers. This concept is already in practice among sellers. For most of the intangible items, sellers share their bad user lists in forums, blogs, social networks and so on. An example of such website is Badbuyerlist [4] where the sellers can get updated list of blacklisted buyers submitted by seller community. Such sharing reduces the risk for collusion and loss in the future by helping sellers in protecting themselves from fraudulent buyers.

Reputation of buyers is not only important in post-paid payments but also in pre-paid ones. For example, when a buyer makes an online transaction using a credit/debit card or third parties, he/she can claim a chargeback mainly for 325 two reasons [45: i) when the credit card number is stolen and used fraudulently and ii) when the buyer bought an item but the seller failed to fulfill his agreements (e.g., did not send the item or it was not as described). This chargeback 
timeframe can vary from weeks and even months after original transaction date. For intangible items, most chargeback claims by buyers are not covered by third parties. Therefore, reputation of buyers by sellers is extremely important for sellers who sell intangible items to protect themselves from future loss and hence to increase the overall profit.

\subsubsection{Experience Calculation}

Experience $\zeta_{x y}$ is calculated by a seller from its trading experiences that include but not limited to payment completion, timely payment and contract completion. This is because although a buyer may complete its payment, it may not be timely and the buyer may also terminate its contract earlier. Therefore, $\zeta_{x y}$ can be defined as:

$$
\zeta_{x y}^{c}(t)=\frac{1}{2} \psi_{x y}(t)\left(s_{x y}^{T}(t)+s_{x y}^{D}(t)\right),
$$

where, $\zeta_{x y}^{c}(t)$ is the current experience; $\psi_{x y}(t)$ is the payment completion func340 tion; $s_{x y}^{T}(t)$ is the timely payment and $s_{x y}^{D}(t)$ is the contract duration function. $\psi_{x y}(t)$ and $s_{x y}^{T}(t)$ can be respectively defined as:

$$
\begin{gathered}
\psi_{x y}(t)= \begin{cases}1 & \text { if completed } \\
0 & \text { otherwise }\end{cases} \\
s_{x y}^{T}(t)=e^{-\beta_{T} i}
\end{gathered}
$$

where, $i$ is the time passed after payment deadline and $\beta_{T}$ is the time sensitivity parameter of timely payment. Therefore, a buyer's trustworthiness is reduced with its delay in payment and $\beta_{T}$ varies with the sellers' business policy. If a seller sets a large value for $\beta_{T}$, the buyer's reputation in terms of timely payment decreases rapidly with the number of overdue days and vice versa. Now, $s_{x y}^{D}(t)$ can be calculated as:

$$
s_{x y}^{D}(t)= \begin{cases}\frac{\tau_{u}}{\tau_{c}} & \text { if } \tau_{u} \leq \tau_{c} \\ 1 & \text { otherwise }\end{cases}
$$


where, $\tau_{c}$ is the contract duration from the seller and $\tau_{u}$ is the active time of the buyer under the contract. Finally, the total experience of a seller can be calculated considering the past experiences using an exponential moving average (EMA) as:

$$
\zeta_{x y}(t)= \begin{cases}\alpha_{e} \zeta_{x y}^{c}(t)+\left(1-\alpha_{e}\right) \zeta_{x y}(t-1) & \text { if } t>1 \\ \zeta_{x y}^{c}(t) & \text { otherwise }\end{cases}
$$

where, $\alpha_{e}$ is a weight factor between 0 and 1 , and $t$ represents a trading episode within a particular window of episodes. Larger weight factors give more importance to the recent experience, whereas smaller values of $\alpha_{e}$ would take past experiences as well. For exponential moving average, $\alpha_{e}$ can be represented in terms of $W$, i.e., $\alpha_{e}=2 /(W+1)$, where, $W$ is the window size. Seller $y$ can forward $\zeta_{x y}(t)$ to others requiring recommendations. The receiving seller can use it in calculating its own reputation value for buyer $x$.

As shown in Fig. 2 a seller can have reliability of a buyer either from a reliability broker or using our proposed model. In case of the latter, a seller can obtain recommendations about a buyer from different sources, e.g., referred recommenders, public social networks, list of blacklisted buyers and other recommenders. The concept of referencing is used in many cases, especially in job applications where the employee can collect and verify information about the applicant from referred recommenders. Public social networks, such as, facebook, twitter, can also become means of collecting recommendations based on text-analysis or page ranks [46]. A seller may also collect information on a prospective buyer from a member of its own loyalty club if the buyer is known to that member. Blacklisted buyers are also a very good source for a seller to know about the fraudulent buyers. Although a seller can collect recommendations from these various sources, it is out of the scope of this paper to explore all possible ways. For ease of calculation and simplicity, in the remaining sections, we assume that a seller will obtain recommendations about a buyer from any such sources, and they are, in general, termed as recommenders. 


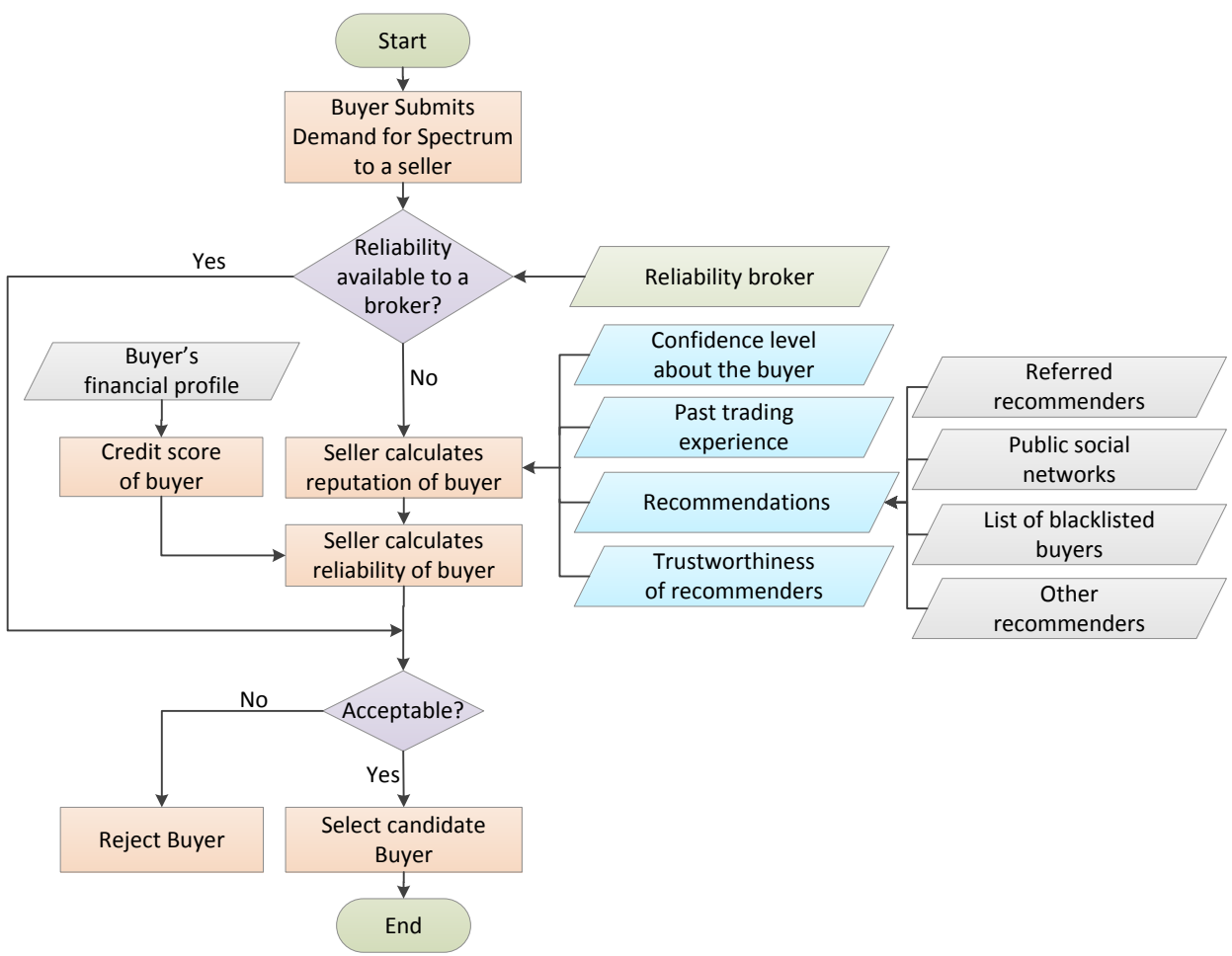

Figure 2: Buyer selection process executed by each seller. 
scores of a recommender, $\phi_{x j}(t)$ can be calculated using EMA as follows:

$$
\phi_{x j}(t)= \begin{cases}\alpha_{e} \phi_{x j}^{c}(t)+\left(1-\alpha_{e}\right) \phi_{x j}(t-1) & \text { if } t>1 \\ \phi_{x j}^{c}(t) & \text { otherwise. }\end{cases}
$$

When a trade ends, the trustworthiness of a recommender will be updated

385 in future trading because $\phi_{x j}$ below a certain predefined value is not considered in the calculation of (3). Fig. 2 shows the candidate buyer selection process of the proposed model.

\section{Price Modelling through Seller Competition}

390 they compete with each other in maximizing their own profits, non-cooperative game theory is an appropriate choice to model this price competition. Therefore, in this paper, we adopted non-cooperative game theory to determine the pricing solution.

In [15], we proposed a market scenario where a buyer considers trading with a potential seller on the expectation of fulfilling certain requirements from the seller in terms of the following metrics: reputation of a seller $y$ to buyer $x, \rho_{x y}$; advertised SNR offered by the seller to the buyer, $\gamma_{x y}$; bandwidth offered for sale, $b_{y}$; available service duration, $\tau_{a_{y}}$ and spectrum price, $p_{x y}$. The perceived reputation $\rho_{x y}$ of seller $y$ can be either obtained from a reputation broker as described in the previous section or the buyer itself can calculate it utilizing a reputation and trustworthiness modelling that employs its own experience [15]. A buyer may not consider sellers if certain requirements are not met, e.g., sellers with reputation and SNR below certain level.

Besides, a buyer may have other requirements to select spectrum from a seller. For example, Weiss et al. 47 mentioned that spectrum is not suitable for fungibility because of the various characteristics of spectrum in spatial and temporal dimensions. Besides, some technological as well as other requirements 
may also impact spectrum fungibility. In [15], we integrated the temporal aspect of fungibility by considering the available service duration, $\tau_{a_{y}}$ of the offered spectrum of seller $y$. Spectrum SNR also includes the technological fungibility in our model. Following [47, below we consider the spatial aspect of spectrum fungibility.

If $f_{x}$ is the required frequency of buyer $x$ and $f_{y}$ is the offered frequency of seller $y$ for bandwidth $b_{y}$, the probabilistic spatial fungibility score for this spectrum is defined as

$$
S_{x y}=\min \left(\frac{f_{x}}{f_{y}}, 1\right)
$$

If $f_{y}<f_{x}$, greater coverage can be achieved with $f_{y}$ [47] and $S_{x y}=1$. Now, a buyer filters out unsuitable sellers and selects only the set of sellers ${ }_{420}$ using buyer-specific requirement criteria, like $\rho_{x y}^{\min } \leq \rho_{x y} \leq \rho_{x y}^{\max }, b_{y} \geq b_{y}^{\min }$, $\gamma_{x y}^{\min } \leq \gamma_{x y} \leq \gamma_{x y}^{\max }, \tau_{a_{y}} \geq \tau_{a_{y}}^{\min }$ and $S_{x y} \geq S_{x y}^{\min }$ that a seller must satisfy. We denote these chosen sellers by buyer $x$ as a set $\Im_{x}$. Buyers request the pricing information to the selected sellers. Once $\Im_{x}$ is determined, we apply the following modelling to determine price through competition among sellers ${ }_{425} y \in \Im_{x}$.

The profit of each primary service provider, i.e., seller can be defined as the revenue earned from serving primary users, the revenue gained by sharing spectrum with secondary users and the cost for sharing the bandwidth. We thus define the supply function (i.e., profit) for primary provider (i.e., seller) $y$ as follows:

$$
\varphi_{b_{y}}(\mathbb{P})=n_{y} \log \left(\frac{\mathscr{B}_{y}-b_{y}}{n_{y}}\right)+\aleph_{x y} p_{x y} b_{y}-b_{y} \mathscr{C}(\mathbb{P}),
$$

where, $\mathscr{C}(\mathbb{P})$ is the cost function of primary service provider; $\mathbb{P}=\left\{p_{x y} \mid y \in \Im_{x}\right\}$ is the set of strategies (i.e., prices) of the selected primary service providers; $n_{y}$ is the number of primary users under a primary service provider; $\mathscr{B}_{y}$ is the total bandwidth of primary provider $y$ and $b_{y}$ is the bandwidth for sale. Incorporating 
the pricing information of other sellers, the cost function $\mathscr{C}(\mathbb{P})$ is defined as 11

$$
\mathscr{C}(\mathbb{P})=k_{1}+k_{2}\left(\sum_{p_{x y} \in \mathbb{P}} p_{x y}\right)^{\alpha_{c}},
$$

where, $k_{1}, k_{2}$ and $\alpha_{c}$ are non-negative constants. Here, $\alpha_{c} \geq 1$ so that the cost function is convex and $0 \leq k_{1}, k_{2} \leq 1$.

For a secondary service provider (i.e., buyer), profit can be defined as the difference between the satisfaction or utility earned from using the bandwidth from a seller and the price paid for the bandwidth. Therefore, the demand function or profit of a buyer can be written as:

$$
\begin{aligned}
\mathscr{P}_{b_{x}}= & n_{x} \rho_{x y} \log \left(\frac{b_{x}}{n_{x}} \log _{2}\left(1+\gamma_{x y}\right)\right) g_{x y}\left(\tau_{a y}, \tau_{r}\right) \\
& -p_{x y} b_{x}
\end{aligned}
$$

where, $b_{x}$ is the bandwidth sought by buyer $x ; n_{x}$ is the number of secondary users under a secondary service provider; $g_{x y}\left(\tau_{a}, \tau_{r}\right)$ is the utility function for the required contract duration and $\tau_{r}$ is the duration required by the secondary provider. The two logarithmic functions are used in (15) for two completely different purposes. The inside $\log ($.$) is used to represent the mean throughput$ using Shannon capacity model defined by $\left(\frac{b_{x}}{n_{x}} \log _{2}\left(1+\gamma_{x y}\right)\right)$. If we omit the inside $\log ($.$) , then the term \frac{b_{x}}{n_{x}}$ indicates mean bandwidth which could also be used instead of the mean throughput. But we prefer to use $\left(\frac{b_{x}}{n_{x}} \log _{2}\left(1+\gamma_{x y}\right)\right)$, i.e., mean throughput, as the parameter of the outer $\log ($.$) function to include$ the channel quality of the bandwidth offered by a seller. On the other hand, the outer $\log ($.$) is used to reflect the diminishing property of marginal utility$ which indicates that the marginal utility decreases as the demand of the product increases. Here, the marginal utility of a product or service represents the change in utility with an increase (or decrease) in the consumption of that product or service [48. Therefore, the outer $\log ($.$) function ensures that the diminishing$ property of marginal utility of economics is reflected. 
Here, $g_{x y}\left(\tau_{a}, \tau_{r}\right)$ can be defined as in [15]:

$$
g_{x y}\left(\tau_{a}, \tau_{r}\right)= \begin{cases}e^{-\beta\left(\tau_{r}-\tau_{a}\right) / \tau_{r}} & \text { if } \tau_{r} \geq \tau_{a} \\ 1 & \text { otherwise }\end{cases}
$$

Differentiating (15) with respect to bandwidth yields marginal demand or ${ }_{460}$ profit function $\left(\mathscr{D}_{b_{x}}\right)$ of the secondary provider. Putting $\frac{\partial \mathscr{P}_{b_{x}}}{\partial b_{x}}=0$ leads to:

$$
\mathscr{D}_{b_{x}}=b_{x}=g_{x y}\left(\tau_{a y}, \tau_{r}\right) \frac{n_{x} \rho_{x y}}{p_{x y}} .
$$

Bandwidth size $b_{y}$ of the profit function (13) can be replaced by the required bandwidth or bandwidth demand $D_{b_{x}}$ of (17). Using (14) and (17), 13) can be rearranged as :

$$
\begin{aligned}
\varphi_{b_{y}}(\mathbb{P})=n_{y} \log \left(\frac{\mathscr{B}_{y}}{n_{y}}-\frac{n_{x} \rho_{x y} g_{x y}\left(\tau_{a y}, \tau_{r}\right)}{n_{y} p_{x y}}\right) \\
+n_{x} \rho_{x y} g_{x y}\left(\tau_{a y}, \tau_{r}\right) \\
\cdot\left(\aleph_{x y}-\frac{k_{1}+k_{2}\left(\sum_{p_{x y} \in \mathbb{P}} p_{x y}\right)^{\alpha_{c}}}{p_{x y}}\right) .
\end{aligned}
$$

Now using $\frac{\partial \varphi_{b y}(\mathbb{P})}{\partial p_{x y}}$,

$$
\begin{aligned}
\frac{\partial \varphi_{b_{y}}(\mathbb{P})}{\partial p_{x y}}= & \frac{n_{y} n_{x} \rho_{x y} g_{x y}\left(\tau_{a y}, \tau_{r}\right)}{p_{x y}\left(\mathscr{B}_{y} p_{x y}-n_{x} \rho_{x y} g_{x y}\left(\tau_{a y}, \tau_{r}\right)\right)} \\
& -\frac{n_{x} \rho_{x y} g_{x y}\left(\tau_{a y}, \tau_{r}\right)}{p_{x y}^{2}} \\
& \cdot\left(p_{x y} \alpha_{c} k_{2}\left(\sum_{p_{x y} \in \mathbb{P}} p_{x y}\right)^{\alpha_{c}-1}\right. \\
& \left.-k_{1}-k_{2}\left(\sum_{p_{x y} \in \mathbb{P}} p_{x y}\right)^{\alpha_{c}}\right) .
\end{aligned}
$$

The Nash equilibrium solution $p_{x y}^{*}$ can be obtained from $(19)$ by solving for all $y$. The spectrum size can then be obtained from $D_{b_{x}}$ for all values 
of $\mathbb{P}^{*}$. It is impractical due to high communication overhead for the primary service providers or sellers to obtain the pricing information and profits of all other sellers. But a seller can observe the adaptation of bandwidth demand by secondary service providers or buyers in response to the seller's pricing strategy. If buyers' demand decreases due to the seller's price increase, it indicates that other sellers' prices are lower. Then, the seller will also decrease the price. This adaptation of price will continue iteratively until the Nash equilibrium price is achieved. Thus, the Nash equilibrium can be obtained for each seller based on the bandwidth demand from buyers. Each seller will try to maximize its profit by adjusting its spectrum pricing based on the marginal profit function of 19 . Therefore, a seller's price adjustment strategy can be modeled as a dynamic game as follows:

$$
p_{x y}(t+1)=p_{x y}(t)+\sigma \frac{\partial \varphi_{b_{y}}(\mathbb{P})}{\partial p_{x y}(t)},
$$

where, $\sigma$ is the learning rate which acts as a weighting factor and its impact on reaching the equilibrium price is detailed in Section 7.2.7. Assuming prices to be the strategies of the players (i.e., sellers), the Nash equilibrium can be obtained by using the best response function which implies the best price of one seller given other's prices. The best response function of seller $y$ (i.e., the best price $p_{x y}$ of seller $y$ ) based on the set of prices $\mathbb{P}_{-y}=\left\{p_{x j} \mid j \neq y\right\}$ offered by other sellers, can be defined as follows:

$$
F_{y}\left(\mathbb{P}_{-y}\right)=\arg \max _{p_{x y}} \varphi_{b_{y}}\left(\mathbb{P}=\left\{p_{x y}\right\} \cup \mathbb{P}_{-y}\right) .
$$

The vector $\mathbb{P}^{*}$ represents the Nash equilibrium solution and can be defined as:

$$
\mathbb{P}^{*}=\left\{p_{x y}^{*} \mid p_{x y}^{*}=F_{y}\left(\mathbb{P}_{-y}^{*}\right), \forall y\right\} .
$$




\section{Bandwidth Trading}

In this paper, we have modeled two types of buyers: (i) price sensitive

490

that maximizes its utility function satisfying its budget constraint or bandwidth requirements.

For the price sensitive buyer, the objective function can be written as:

Determine $\mathbf{b}$

Maximize

$$
\begin{aligned}
u_{x}(\mathbf{b})= & \sum_{y \in \Im_{x}} n_{x} \rho_{x y} \log \left(\frac{b_{y}}{n_{x}} \log _{2}\left(1+\gamma_{x y}\right)\right) \\
& . e^{-\beta\left(\tau_{r}-\tau_{a_{y}}\right) / \tau_{r}}
\end{aligned}
$$

subject to

$$
\sum_{y \in \Im_{x}} p_{x y}^{*} b_{y} \leq I_{x}
$$

where, $\mathbf{b}$ is the set of bandwidths a buyer wants to buy; $\beta$ is a constant which was numerically derived in [15, and $I_{x}$ is the budget constraint.

We can solve 23 Appendix A for details) to find the optimized amount of bandwidth $b_{y}$ for the price sensitive buyer to buy from seller $y$ to maximize its objective function:

$$
b_{y}=\frac{I_{x} \rho_{x y} e^{-\beta\left(\tau_{r}-\tau_{a_{y}}\right) / \tau_{r}}}{p_{x y} \sum_{y \in \Im_{x}} \rho_{x y} e^{-\beta\left(\tau_{r}-\tau_{a_{y}}\right) / \tau_{r}}} .
$$

If a buyer is not price sensitive (i.e., not having constraint on budget), it will focus on obtaining its required bandwidth of higher quality from highly 
reputable sellers. For price insensitive buyers, the problem can be formulated as:

$$
\begin{aligned}
& \text { Determine } \mathbf{b} \\
& \text { Maximize } \\
& \qquad \begin{aligned}
u_{x}(\mathbf{b})= & \sum_{y \in \Im_{x}} n_{x} \rho_{x y} \log \left(\frac{b_{y}}{n_{x}} \log _{2}\left(1+\gamma_{x y}\right)\right) \\
& . e^{-\beta\left(\tau_{r}-\tau_{a_{y}}\right) / \tau_{r}}
\end{aligned}
\end{aligned}
$$

subject to

$$
\sum_{y \in \Im_{x}} b_{y}=\mathscr{R}_{x}
$$

where, $\mathscr{R}_{x}$ is the total bandwidth requirement.

In a similar way adopted for (23), we can also find the optimum amount of bandwidth $b_{y}$ to buy from seller $y$ for a price insensitive buyer by solving [25):

$$
b_{y}=\frac{\mathscr{R}_{x} n_{x} \rho_{x y} e^{-\beta\left(\tau_{r}-\tau_{a_{y}}\right) / \tau_{r}}}{\sum_{y \in \Im_{x}} \rho_{x y} e^{-\beta\left(\tau_{r}-\tau_{a_{y}}\right) / \tau_{r}}} .
$$

Based on the Nash equilibrium pricing information $\mathbb{P}^{*}$ obtained in 22, buyers, such as price sensitive buyers, will calculate analytically the amount of bandwidth it should buy from seller(s) within its budget constraint using (24). Similarly, price insensitive buyers will select the seller(s) to buy from and the corresponding bandwidth size from each using (26).

Although optimum bandwidth is obtained using the analytical solutions in (24) and (26), the calculated bandwidth from a seller may be less than the 515 minimum amount a buyer want to buy from any seller, i.e., $b_{y} \leq b_{y}^{\min }$. In that case, a buyer will calculate bandwidth from the remaining sellers and start trading.

Algorithm 1 describes the steps involving in the proposed spectrum trading model. The local stability analysis of the model is provided in Appendix B. 


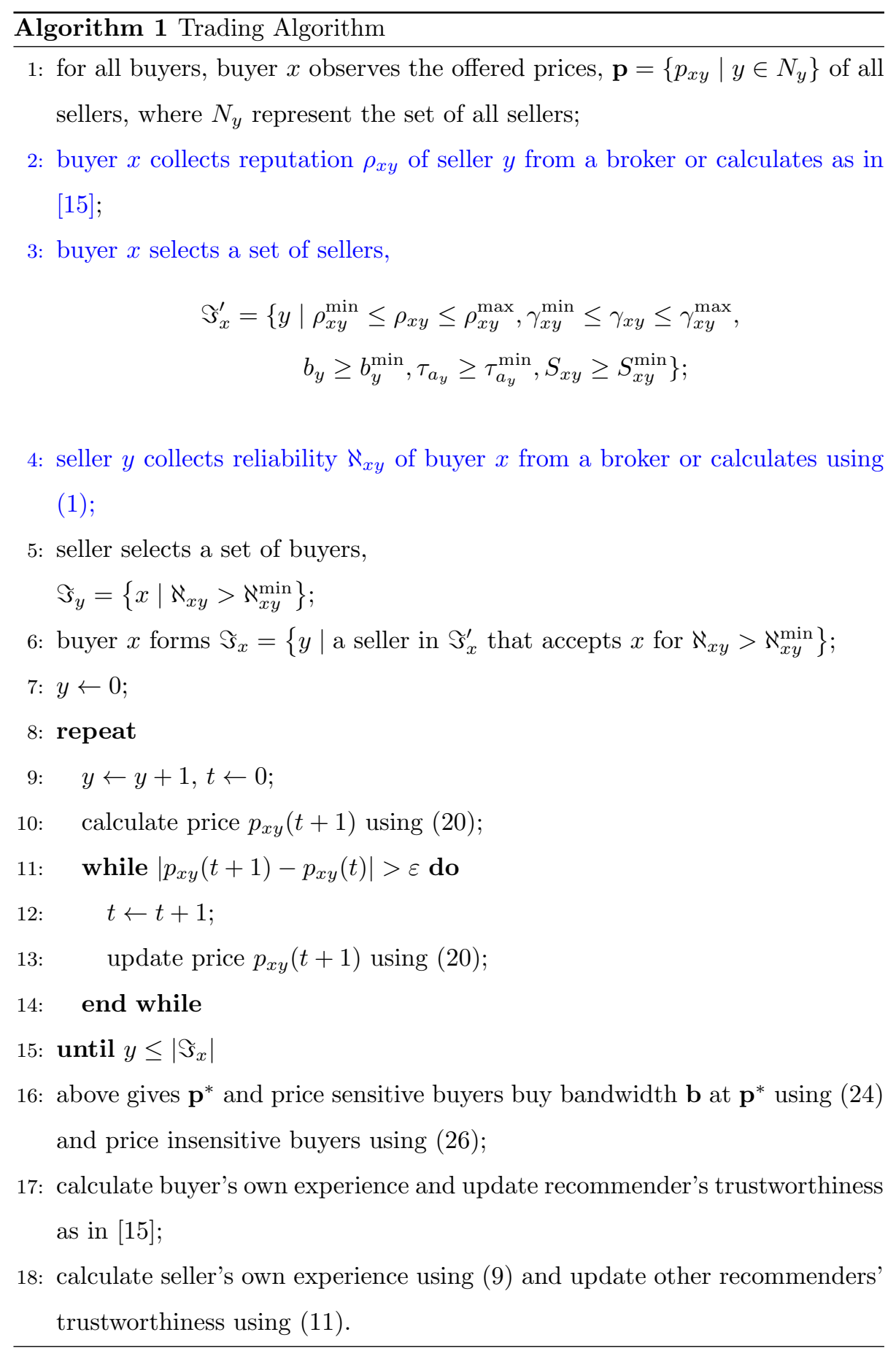




\section{Performance Evaluation}

\subsection{Experimental Setup}

Performance evaluation and numerical simulation for comparative analyses have been carried out using Matlab R2012b. We have considered a dynamic spectrum sharing scenario with 10 primary service providers (sellers) each hav-

525 ing a bandwidth of $20 \mathrm{MHz}$ and 20 secondary service providers (buyers). Each of the primary and secondary services are considered to have 10 primary and 10 secondary users, respectively. Results are obtained from the average of 100 runs for each individual primary services. Reputation of the primary services is considered to be in the range from 0.1 to 1 and SNR is assumed to be from 1 to $35 \mathrm{~dB}$. In our simulation, reliability of a buyer or reputation of a seller is taken from a broker with probability 0.4 , i.e., with probability 0.6 , it is calculated by the entity itself using our model.

\subsection{Numerical Results}

\subsubsection{Best Response and Nash Equilibrium}

As a representative example, the best response of two primary providers or sellers is shown in Fig. 3. However, all the results are using 10 sellers. The best response of one seller is a function of the other's strategy (e.g., price setting). In other words, the best response is the best price $p_{1,1}$ of Seller 1 when this seller knows the best price $p_{1,2}$ of Seller 2 (both offering price to Buyer 1), and vice versa. Therefore, the Nash equilibrium is found where the two best responses intersect. If the price of one seller increases, the price of the other seller also increases. Evidently, the price of one seller impacts the price of another seller which implies that in a competitive market, a seller's profit depends on that of other sellers as well. Similar results are observed for multiple sellers.

\subsubsection{Marginal Profit}

Figure 4 shows the impact of seller's reputation $\rho_{x y}$, available contract duration $\tau_{a y}$ and $\alpha_{c}$ (constant related to the cost function defined in (14)) on the 
Figure 4: Marginal profit of sellers with different values of reputation, available contract duration and $\alpha_{c}$.

marginal profit of sellers. Marginal profit increases for higher values of reputation because a higher reputation creates more demand from buyers. Similarly, longer available contract duration creates higher demand from buyers. With increasing $\alpha_{c}$, marginal profit increases but for $\alpha_{c}$ above 0.8 , profit starts to decline.

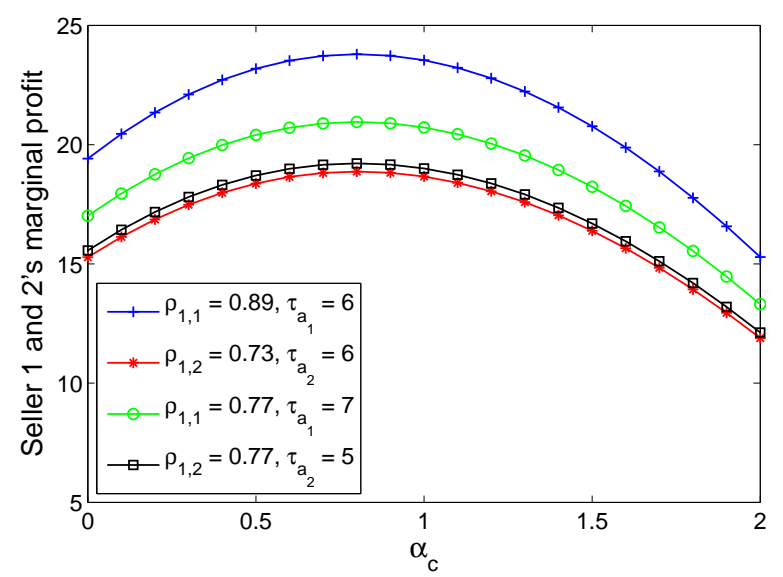




\subsubsection{Buyer's Reputation and Trustworthiness}

As mentioned before, a seller's profit depends on its buyers' reputation if depends on the confidence level of seller's experiences, other recommenders' recommendations, buyer's past payment completion status, timely payments and contract durations. Then, seller's normalized profit is calculated using (19) which is shown in Fig. 5 . It reveals that for leasing based contracts, selecting a reputed buyer is also important for a seller.

Fig. 6] shows a comparison of seller's profit with and without considering buyer's reliability. In [15, we considered only the seller's reputation. In this paper, we consider buyer's credit scoring and reputation to determine its reliability in order to reduce the loss of profit of a seller. It is observed from the figure that if buyers' reliability is considered, a seller's profit is increased. However, if buyers' reliability is not considered (as in [15]), a seller may choose a less reliable buyer, and therefore, makes less profit due to an incomplete payment or termination of the contract. 


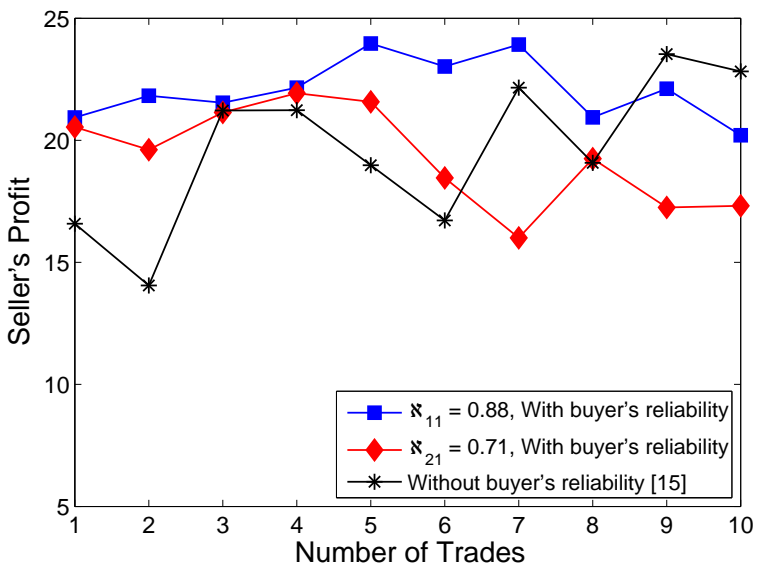

Figure 6: Seller's profit comparison with and without reliability of buyers.

\subsubsection{Throughput Calculation}

Assuming additive white Gaussian noise (AWGN) at the receiver, throughput can be defined as the number of payload bits per second received correctly [49]:

$$
\text { Throughput, } \Psi=\frac{K R}{L}\left(1-\frac{1}{2} e^{-\gamma / 2}\right)
$$

where, $L$ is the packet size in bits; $R$ is the transmission rate in bits/sec and $K$ is the payload size in bits.

Advertised SNR or the initial SNR may not prevail throughout the whole trading period. Since our reputation model is built upon the consistency of the received SNR which is measured from the buyer's own and recommenders' trading experience, a buyer tends to select a reliable seller for which the throughput will be higher. Therefore, to assess the impact of the seller's reputation, average throughput is calculated using (27) and compared between the proposed model and MCCPSS (market-equilibrium, competitive and cooperative pricing for spectrum sharing) [17] which is shown in Fig. 7. Our game theoretic modelling of spectrum trading has been compared to MCCPSS [17] because MCCPSS also uses game theory in pricing solution. Since reputation is not incorporated in MCCPSS, buyers may select a seller with inconsistent SNR or whose channel may become congested during the trading period resulting 


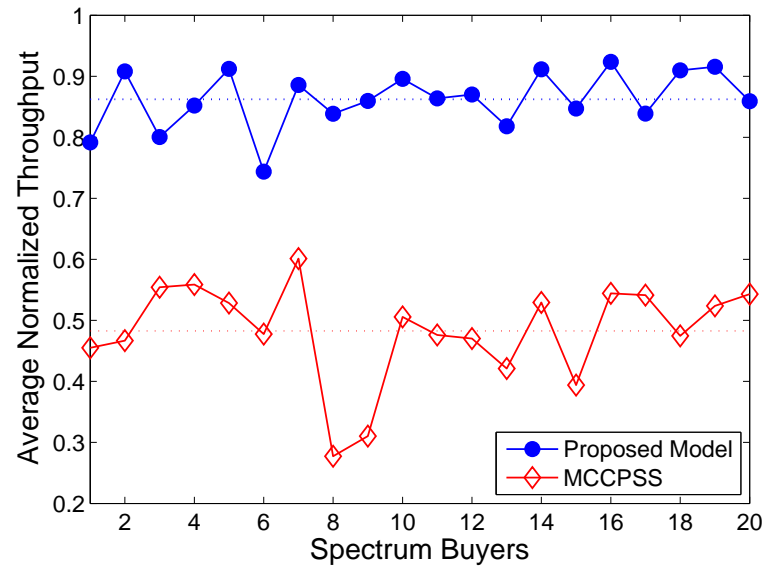

Figure 7: Impact of seller's reputation on average throughput of the spectrum buyers.

in lower throughput, whereas the proposed model assists the buyers to select sellers with consistent SNR, ensuring higher average throughput.

Fig. 8 shows a comparison of the profit or benefit of spectrum buyers in terms of the ratio of Nash equilibrium price to throughput between the proposed model and MCCPSS [17. The randomness in the ratio output of this figure is due to the variations in the obtained Nash equilibrium prices from different sellers. The price to throughput ratio is always less in our proposed model, which implies that our proposed model provides more benefit to the buyers. Since the spectrum buyers in our model select higher quality spectrum from highly demanded sellers, it results in higher throughput.

\subsubsection{Price Sensitive and Insensitive Buyers}

Table 1 shows the traded bandwidth of price sensitive buyers from different sellers with different requirements. It can be observed from the table that Buyer 1 's maximum limit of reputation requirement is 1 whereas it is 0.8 for Buyer 2. Buyer 2's other requirements are similar to the requirements of Buyer 1. Since Buyer 2 has selected less requirement for reputation, it can purchase more bandwidth (7.44 vs $7.15 \mathrm{MHz}$ ) with the same budget constraint because of less price per unit bandwidth for less reputed seller. Buyer 3's budget requirement 


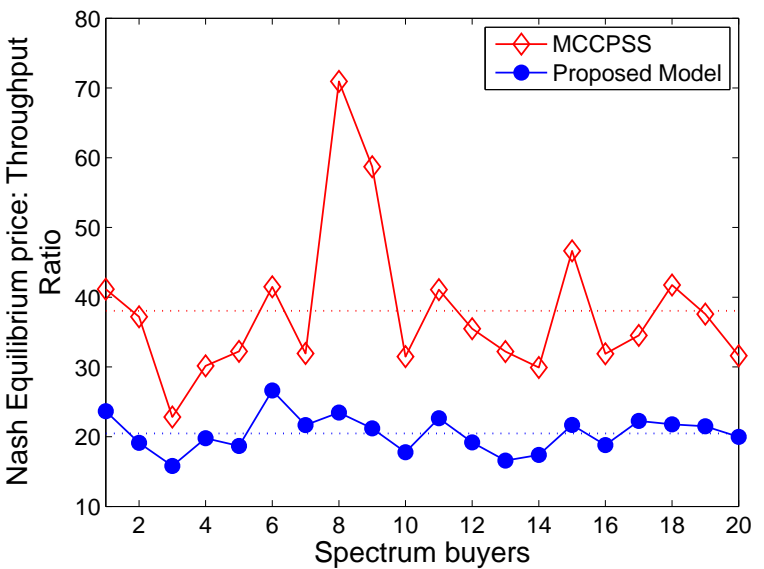

Figure 8: The ratio of Nash equilibrium price to throughput between the proposed model and MCCPSS [17 for different users.

is less than Buyers 1 and 2, therefore, Buyer 3 purchases less bandwidth than the others. In each case, within the budget constraint, a buyer buys more bandwidth from a reputed seller than a less reputed seller.

Traded bandwidth for price insensitive buyers is shown in Table 2. Buyer 5 sets an upper limit for reputation at 0.8, less than that of Buyer 4. Therefore, Buyer 5 does not select a seller with reputation greater than 0.8 , e.g., 0.9 , as shown in Table 2. Then Buyer 5 can buy the same total bandwidth of $10 \mathrm{MHz}$ for a lower price (20.30 vs 21.14 price unit) compared to Buyer 4. Thus, a buyer can have different types of filtering requirements based on its policy in selecting sellers to buy more bandwidth or spend less in buying the spectrum resources.

\subsubsection{Profit Comparison}

Figure 9 illustrates the profit of sellers by serving own primary users and sharing spectrum with the secondary providers or buyers. If the price per unit bandwidth is increased, the profit of the seller or primary service provider also increases and then tends to become saturated due to the lower demand from buyers. The Nash equilibrium was found where both the profits intersect.

The sellers with higher reputation have higher demands from buyers. The increased demand also causes the price of the product to increase resulting in 
Table 1: Traded bandwidth of price sensitive buyers

\begin{tabular}{|c|c|c|c|c|c|c|c|c|}
\hline \multicolumn{9}{|c|}{ Price Sensitive Buyer 1} \\
\hline \multirow{2}{*}{$I_{x}$} & \multirow{2}{*}{$\begin{array}{c}\text { Seller Selection } \\
\text { Criteria }\end{array}$} & \multicolumn{5}{|c|}{ Selected Sellers } & \multirow{2}{*}{$\begin{array}{l}\text { Purchased } \\
\text { Bandwidth }\end{array}$} & \multirow{2}{*}{ Spent } \\
\hline & & $\rho_{1 y}$ & $\gamma_{1 y}$ & $\tau_{a_{y}}$ & $p_{1 y}$ & $S_{1 y}$ & & \\
\hline \multirow{4}{*}{15} & \multirow{4}{*}{$\begin{array}{c}0.5 \leq \rho_{1 y} \leq 1 \\
22 \leq \gamma_{1 y} \leq 35 \\
\tau_{a_{y}} \geq 7, S_{1 y} \geq 0.7\end{array}$} & 0.9 & 32 & 36 & 2.3 & 0.9 & 2.02 & 4.66 \\
\hline & & 0.8 & 29 & 27 & 2.2 & 1.0 & 1.88 & 4.14 \\
\hline & & 0.7 & 28 & 34 & 2.0 & 1.0 & 1.81 & 3.62 \\
\hline & & 0.5 & 26 & 25 & 1.8 & 0.9 & 1.44 & 2.59 \\
\hline \multicolumn{7}{|c|}{ Total } & 7.15 & 15 \\
\hline
\end{tabular}

\begin{tabular}{|c|c|c|c|c|c|c|c|c|}
\hline \multicolumn{9}{|c|}{ Price Sensitive Buyer 2} \\
\hline \multirow{2}{*}{$I_{x}$} & \multirow{2}{*}{$\begin{array}{c}\text { Seller Selection } \\
\text { Criteria }\end{array}$} & \multicolumn{5}{|c|}{ Selected Sellers } & \multirow{2}{*}{$\begin{array}{l}\text { Purchased } \\
\text { Bandwidth }\end{array}$} & \multirow{2}{*}{ Spent } \\
\hline & & $\rho_{2 y}$ & $\gamma_{2 y}$ & $\tau_{a_{y}}$ & $p_{2 y}$ & $S_{2 y}$ & & \\
\hline \multirow{3}{*}{15} & \multirow{3}{*}{$\begin{array}{c}0.5 \leq \rho_{2 y} \leq 0.8 \\
22 \leq \gamma_{2 y} \leq 35 \\
\tau_{a_{y}} \geq 7, S_{2 y} \geq 0.7\end{array}$} & 0.8 & 29 & 27 & 2.2 & 0.9 & 2.73 & 6.00 \\
\hline & & 0.7 & 28 & 34 & 2.0 & 1.0 & 2.63 & 5.25 \\
\hline & & 0.5 & 26 & 25 & 1.8 & 1.0 & 2.08 & 3.75 \\
\hline \multicolumn{7}{|c|}{ Total } & 7.44 & 15 \\
\hline
\end{tabular}

\begin{tabular}{|c|c|c|c|c|c|c|c|c|}
\hline \multicolumn{9}{|c|}{ Price Sensitive Buyer 3} \\
\hline \multirow{2}{*}{$I_{x}$} & \multirow{2}{*}{$\begin{array}{c}\text { Seller Selection } \\
\text { Criteria }\end{array}$} & \multicolumn{5}{|c|}{ Selected Sellers } & \multirow{2}{*}{$\begin{array}{l}\text { Purchased } \\
\text { Bandwidth }\end{array}$} & \multirow{2}{*}{ Spent } \\
\hline & & $\rho_{3 y}$ & $\gamma_{3 y}$ & $\tau_{a_{y}}$ & $p_{3 y}$ & $S_{3 y}$ & & \\
\hline \multirow{3}{*}{12} & \multirow{3}{*}{$\begin{array}{c}0.5 \leq \rho_{3 y} \leq 0.8 \\
20 \leq \gamma_{3 y} \leq 30 \\
\tau_{a_{y}} \geq 4, S_{3 y} \geq 0.8\end{array}$} & 0.8 & 29 & 27 & 2.2 & 1.0 & 2.18 & 4.80 \\
\hline & & 0.7 & 28 & 34 & 2.0 & 0.9 & 2.10 & 4.20 \\
\hline & & 0.5 & 26 & 25 & 1.8 & 0.8 & 1.67 & 3.00 \\
\hline \multicolumn{7}{|c|}{ Total } & 5.95 & 12 \\
\hline
\end{tabular}

${ }_{620}$ more profits for the seller. In Fig. 10 the profit of a primary service provider (i.e., seller) is calculated with an increasing order of reputation. Since higher reputation causes higher demand, a highly reputed seller earns higher profit 
Table 2: Traded bandwidth of price insensitive buyers

\begin{tabular}{|c|c|c|c|c|c|c|c|c|}
\hline \multicolumn{9}{|c|}{ Price Insensitive Buyer 4} \\
\hline \multirow{2}{*}{$\mathscr{R}_{x}$} & \multirow{2}{*}{$\begin{array}{c}\text { Seller Selection } \\
\text { Criteria }\end{array}$} & \multicolumn{5}{|c|}{ Selected Sellers } & \multirow{2}{*}{$\begin{array}{l}\text { Purchased } \\
\text { Bandwidth }\end{array}$} & \multirow{2}{*}{ Spent } \\
\hline & & $\rho_{4 y}$ & $\gamma_{4 y}$ & $\tau_{a_{y}}$ & $p_{4 y}$ & $S_{4 y}$ & & \\
\hline \multirow{4}{*}{10} & \multirow{4}{*}{$\begin{array}{c}0.5 \leq \rho_{4 y} \leq 1 \\
25 \leq \gamma_{4 y} \leq 35 \\
\tau_{a_{y}} \geq 7, S_{4 y} \geq 0.8\end{array}$} & 0.9 & 32 & 36 & 2.3 & 0.9 & 3.10 & 7.14 \\
\hline & & 0.8 & 29 & 27 & 2.2 & 0.9 & 2.76 & 6.07 \\
\hline & & 0.7 & 28 & 34 & 2.0 & 1.0 & 2.41 & 4.83 \\
\hline & & 0.5 & 26 & 25 & 1.8 & 1.0 & 1.72 & 3.10 \\
\hline \multicolumn{7}{|c|}{ Total } & 10 & 21.14 \\
\hline
\end{tabular}

\begin{tabular}{|c|c|c|c|c|c|c|c|c|}
\hline \multicolumn{9}{|c|}{ Price Insensitive Buyer 5} \\
\hline \multirow{2}{*}{$\mathscr{R}_{x}$} & \multirow{2}{*}{$\begin{array}{c}\text { Seller Selection } \\
\text { Criteria }\end{array}$} & \multicolumn{5}{|c|}{ Selected Sellers } & \multirow{2}{*}{$\begin{array}{l}\text { Purchased } \\
\text { Bandwidth }\end{array}$} & \multirow{2}{*}{ Spent } \\
\hline & & $\rho_{5 y}$ & $\gamma_{5 y}$ & $\tau_{a_{y}}$ & $p_{5 y}$ & $S_{5 y}$ & & \\
\hline \multirow{3}{*}{10} & \multirow{3}{*}{$\begin{array}{c}0.5 \leq \rho_{5 y} \leq 0.8 \\
24 \leq \gamma_{5 y} \leq 32 \\
\tau_{a_{y}} \geq 5, S_{5 y} \geq 0.9\end{array}$} & 0.8 & 29 & 27 & 2.2 & 1.0 & 4.0 & 8.80 \\
\hline & & 0.7 & 28 & 34 & 2.0 & 1.0 & 3.5 & 7.00 \\
\hline & & 0.5 & 26 & 25 & 1.8 & 1.0 & 2.5 & 4.50 \\
\hline \multicolumn{7}{|c|}{ Total } & 10 & 20.30 \\
\hline
\end{tabular}

which is shown in this figure. In comparison, a buyer selects a seller in MCCPSS without consideration of reputation. Therefore, highly reputed sellers may earn lower profits than other sellers due to the absence of any reputation system in MCCPSS.

Caicedo and Weiss [50] found in their study that spectrum market is not viable with few numbers of buyers and sellers such as less than five. However, their observation was based on the spectrum auction market. In auction, the market is successful for higher number of buyers and consequently with fewer buyers, it is less viable. But our approach in this paper is different from the auction market. In our model, price is based on supply and demand, and equilibrium is reached based on the price adaptation dynamically, and therefore, 


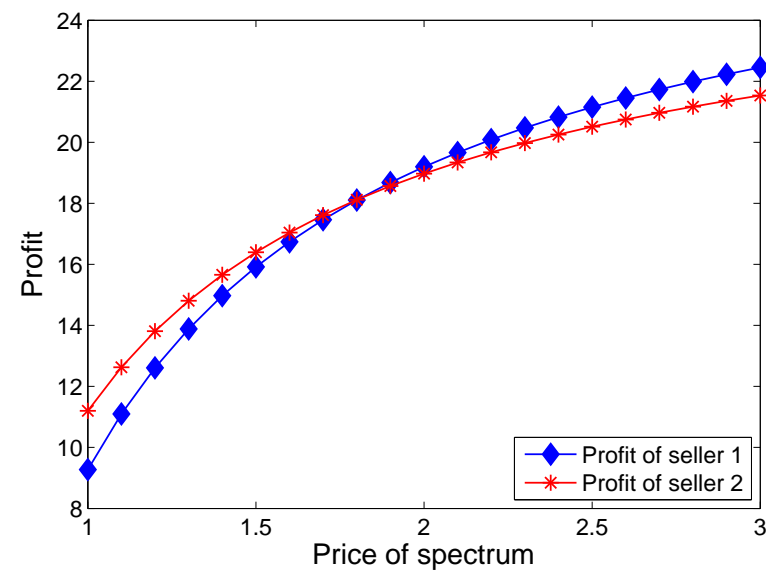

Figure 9: Profit of sellers for different values of price.

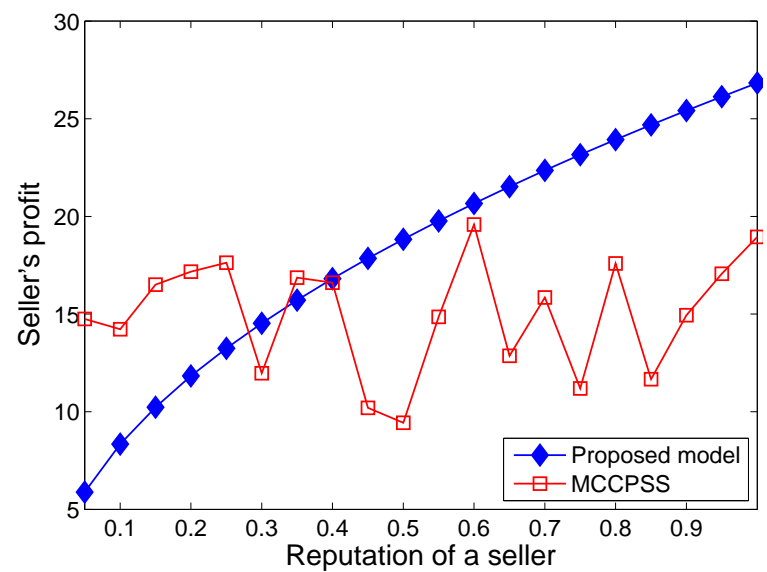

Figure 10: Profit comparison of a seller between the proposed model and MCCPSS [17, respectively.

is applicable for any number of trading partners. Also the number of buyers and sellers in many countries are more than 10 (such as in India [51]), and this number is expected to grow in future due to ever increasing bandwidth demand.

\subsubsection{Stability Analysis}

Learning rate can affect the convergence to Nash equilibrium price which is shown in the local bifurcation analysis [52] of Fig. 11. If the learning rate ${ }_{640}$ is small, the algorithm can reach a unique Nash equilibrium price. For this 


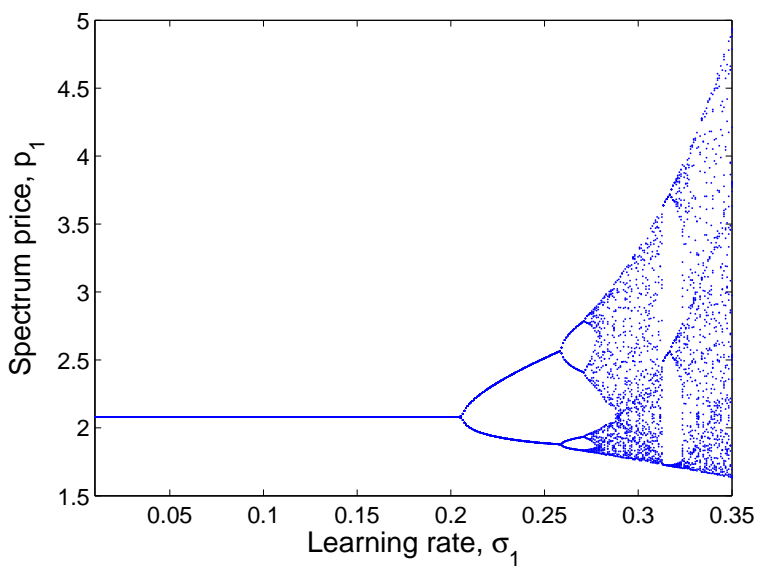

Figure 11: Bifurcation diagram of seller 1 showing the equilibrium price with different learning rates.

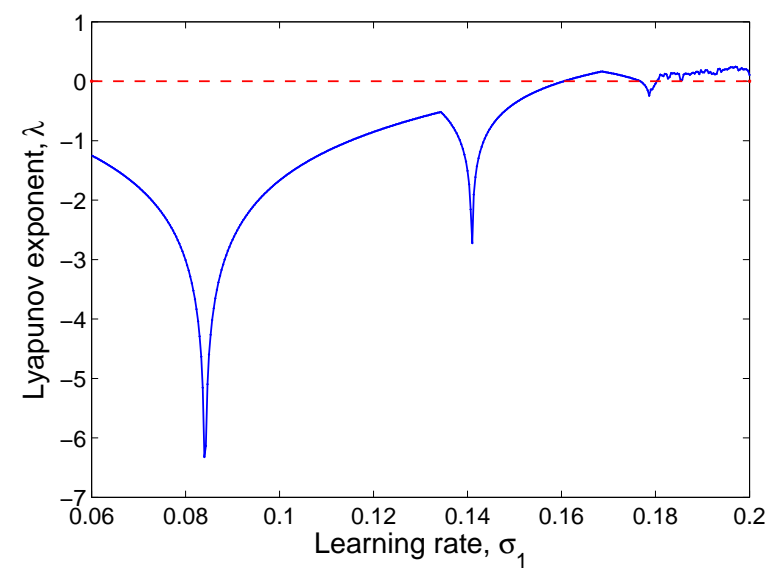

Figure 12: Lyapunov exponent and learning rate.

particular experiment, learning rate $\sigma_{1}>0.22$ exhibits that price fluctuates between two prices. If the learning rate is increased further, the price varies in multiple values and never converges to a single equilibrium value.

Stability of a system in terms of chaos can also be analyzed using the Lyapunov exponent [53]. A positive Lyapunov exponent indicates chaos and it determines the average exponential rate of separation of two nearby initial conditions, or the average stretching of the space. Considering two nearby initial 
points at $p_{0}$ and $p_{0}+\delta p_{0}$ in $p_{n+1}=f\left(p_{n}\right)$, the points are separated after one iteration by:

$$
\delta p_{1}=f\left(p_{0}+\delta p_{0}\right)-f\left(p_{0}\right) \simeq \delta p_{0} f^{\prime}\left(p_{0}\right) .
$$

Local Lyapunov exponent $\lambda$ at $p_{0}$ can be defined as:

$$
\begin{gathered}
e^{\lambda}=\left|\delta p_{1} / \delta p_{0}\right|, \\
\lambda=\ln \left|\delta p_{1} / \delta p_{0}\right| \simeq \ln \left|f^{\prime}\left(p_{0}\right)\right| .
\end{gathered}
$$

Global Lyapunov exponent can then be found by:

$$
\lambda=\lim _{N \rightarrow \infty} \frac{1}{N} \sum_{n=0}^{N-1} \ln \left|f^{\prime}\left(p_{n}\right)\right| .
$$

Figure 12 shows the Lyapunov exponent for different values of the learning rate $\sigma_{1}$ with the adaptation of price. For small values of $\sigma_{1}$, the system reaches the equilibrium because the value of $\lambda$ remains less than 0 . When $\lambda \geq 0$ for 655 large values of $\sigma_{1}$, the system indicates instability or chaos.

\section{Conclusion}

In this paper, we propose a spectrum trading model for cognitive radio networks where there are multiple sellers and multiple buyers. Competition among sellers is modeled using game theory. The Nash equilibrium is obtained dynamically to find the optimal price of the spectrum for each seller. Sellers dynamically adjust their pricing strategy based on the marginal profit calculated from the demands of buyers. Sellers are also able to select suitable buyers based on buyers' creditworthiness and reputation in order to secure long term trading contracts. Reputation/reliability of sellers or buyers can be obtained from reputation brokerage service or calculated using our model. Using the Nash equilibrium price, price sensitive and insensitive buyers calculate the optimum amount of spectrum to buy. Buyers also employ reputation as a criterion 
to evaluate sellers and use recommendations from other buyers to find the best seller(s) in order to maximize throughput. Stability of the dynamic game used by the sellers is analyzed using local stability theory. Experimental results reveal that our proposed model performs better both in producing higher profits for the reputed sellers and higher throughput and lower price to throughput ratio for buyers.

\section{Appendix A. Optimum Bandwidth Considering Price Sensitivity}

To obtain an analytical solution of 23$]$, it can be rewritten using a Lagrange multiplier,

$$
\begin{aligned}
u\left(\mathbf{b}, \lambda_{1}\right)= & \sum_{y \in \Im_{x}} n_{x} \rho_{x y} \log \left(\frac{b_{y}}{n_{x}} \log _{2}\left(1+\gamma_{y}\right)\right) \\
& . e^{-\beta\left(\tau_{r}-\tau_{a y}\right) / \tau_{r}}-\lambda_{1}\left(\sum_{y \in \Im_{x}} p_{x y} b_{y}-I_{x}\right) .
\end{aligned}
$$

Other related constraints on meeting the minimum requirements as discussed earlier are considered to filter out unsuitable sellers before optimization. Now, $\left(\mathbf{b}, \lambda_{1}\right)$ is stationary for $u\left(\mathbf{b}, \lambda_{1}\right)$ only if $\nabla_{\mathbf{b}, \lambda_{1}} u\left(\mathbf{b}, \lambda_{1}\right)=0$. Setting this gradient 680 equal to zero yields

$$
\begin{aligned}
\frac{\partial u}{\partial b_{y}} & =\frac{1}{b_{y}} n_{x} \rho_{x y} e^{-\beta\left(\tau_{r}-\tau_{a_{y}}\right) / \tau_{r}}-\lambda_{1} p_{x y}=0, \\
\frac{\partial u}{\partial \lambda_{1}} & =\sum_{y \in \Im_{x}} p_{x y} b_{y}-I_{x}=0 .
\end{aligned}
$$

Using A.2,

$$
b_{y}=\frac{1}{\lambda_{1} p_{x y}} n_{x} \rho_{x y} e^{-\beta\left(\tau_{r}-\tau_{a_{y}}\right) / \tau_{r}}
$$

From A.3,

$$
\sum_{y \in \Im_{x}} p_{x y} b_{y}=I_{x}
$$


which leads to,

$$
\lambda_{1}=\frac{n_{x}}{I_{x}} \sum_{y \in \Im_{x}} \rho_{x y} e^{-\beta\left(\tau_{r}-\tau_{a_{y}}\right) / \tau_{r}} .
$$

Using A.4 and A.6,

$$
b_{y}=\frac{I_{x} \rho_{x y} e^{-\beta\left(\tau_{r}-\tau_{a_{y}}\right) / \tau_{r}}}{p_{x y} \sum_{y \in \Im_{x}} \rho_{x y} e^{-\beta\left(\tau_{r}-\tau_{a_{y}}\right) / \tau_{r}}},
$$

where, $b_{y}$ is the optimized amount of bandwidth to buy from seller $y$ to maximize its objective function.

${ }_{685}$ Eq. 25 can be similarly solved to calculate the optimum bandwidth for price insensitive buyer.

\section{Appendix B. Local Stability Analysis}

A fixed point in the solution space is stable if the eigenvalues of the Jacobian matrix are strictly inside the unit circle [11, 14]. Here, we can determine the stability at the fixed point of equilibrium price $p_{x y}$ from 20 . Here, we define the Jacobian matrix of two primary services as:

$$
\begin{aligned}
J & =\left[\begin{array}{ll}
\frac{\partial p_{1}[t+1]}{\partial p_{1}[t]} & \frac{\partial p_{1}[t+1]}{\partial p_{2}[t]} \\
\frac{\partial p_{2}[t+1]}{\partial p_{1}[t]} & \frac{\partial p_{2}[t+1]}{\partial p_{2}[t]}
\end{array}\right] \\
& =\left[\begin{array}{ll}
J_{11} & J_{12} \\
J_{21} & J_{22}
\end{array}\right],
\end{aligned}
$$


where,

$$
\begin{aligned}
& J_{11}=1-\sigma_{1} n_{1} \rho_{1} g_{x y}\left[\frac{m_{1}\left(2 \mathscr{B}_{1} p_{1}-n_{1} \rho_{1} g_{x y}\right)}{\left(\mathscr{B}_{1} p_{1}^{2}-n_{1} \rho_{1} g_{x y} p_{1}\right)^{2}}\right. \\
& +\frac{\alpha(\alpha-1) k_{2}\left(\sum_{p_{j} \in \mathbb{P}} p_{j}\right)^{\alpha-2}}{p_{1}} \\
& -\frac{2 \alpha k_{2}\left(\sum_{p_{j} \in \mathbb{P}} p_{j}\right)^{\alpha-1}}{p_{1}^{2}} \\
& \left.+\frac{2\left(k_{1}+k_{2}\left(\sum_{p_{j} \in \mathbb{P}} p_{j}\right)^{\alpha}\right)}{p_{1}^{3}}\right] \\
& J_{12}=\frac{1}{p_{1}^{2}} \sigma_{1} n_{1} \rho_{1} g_{x y} \alpha k_{2}\left(2 p_{1}-\alpha p_{1}+p_{2}\right)\left(\sum_{p_{j} \in \mathbb{P}} p_{j}\right)^{\alpha-2}, \\
& J_{21}=\frac{1}{p_{2}^{2}} \sigma_{2} n_{2} \rho_{2} g_{x y} \alpha k_{2}\left(2 p_{2}-\alpha p_{2}+p_{1}\right)\left(\sum_{p_{j} \in \mathbb{P}} p_{j}\right)^{\alpha-2}, \\
& J_{22}=1-\sigma_{2} n_{2} \rho_{2} g_{x y}\left[\frac{m_{2}\left(2 \mathscr{B}_{2} p_{2}-n_{2} \rho_{2} g_{x y}\right)}{\left(\mathscr{B}_{2} p_{2}^{2}-n_{2} \rho_{2} g_{x y} p_{2}\right)^{2}}\right. \\
& +\frac{\alpha(\alpha-1) k_{2}\left(\sum_{p_{j} \in \mathbb{P}} p_{j}\right)^{\alpha-2}}{p_{2}} \\
& -\frac{2 \alpha k_{2}\left(\sum_{p_{j} \in \mathbb{P}} p_{j}\right)^{\alpha-1}}{p_{2}^{2}} \\
& \left.+\frac{2\left(k_{1}+k_{2}\left(\sum_{p_{j} \in \mathbb{P}} p_{j}\right)^{\alpha}\right)}{p_{2}^{3}}\right] .
\end{aligned}
$$

Since the matrix is neither diagonal nor triangular, eigenvalues of this matrix can be calculated from the characteristic equation which can be written as: ${ }_{690} \lambda^{2}-\lambda\left(J_{11}+J_{22}\right)+\left(J_{11} J_{22}-J_{12} J_{21}\right)=0$. Therefore, the eigenvalues of this matrix are:

$$
\left(\lambda_{1}, \lambda_{2}\right)=\frac{\left(J_{11}+J_{22}\right) \pm \sqrt{\left(J_{11}-J_{22}\right)^{2}+4 J_{12} J_{21}}}{2} .
$$

A fixed point $p_{x y}$ is stable if and only if the eigenvalues $\left(\lambda_{1}, \lambda_{2}\right)$ are all inside the unit circle of the complex plane (i.e., $\left|\lambda_{i}\right|<1$ ). 


\section{References}

${ }_{695}$ [1] F. F. Digham, Joint power and channel allocation for cognitive radios, in: Proceedings of IEEE WCNC, 2008, pp. 882-887.

[2] Spectrum policy task force, FCC, ET Docket No. 02-135 (2002).

[3] E. Hossain, D. Niyato, Z. Han, Dynamic spectrum access and management in cognitive radio networks, Cambridge University Press, 2009.

700 [4] M. B. Weiss, Spatio-temporal spectrum holes and the secondary user, in: Proceedings of IEEE New Frontiers in Dynamic Spectrum Access Networks (DySPAN), 2011, pp. 216-222.

[5] J. M. Peha, Sharing spectrum through spectrum policy reform and cognitive radio, Proceedings of the IEEE 97 (4) (2009) 708-719.

[6] V. Rodriguez, K. Moessner, R. Tafazolli, Auction driven dynamic spectrum allocation: Optimal bidding, pricing and service priorities for multi-rate, multi-class CDMA, in: Proceedings of IEEE PIMRC, Vol. 3, 2005, pp. $1850-1854$.

[7] Y. Xing, R. Chandramouli, C. M. Cordeiro, Price dynamics in competitive agile spectrum access markets, IEEE Journal on Selected Areas in Communications 25 (3) (2007) 613-621.

[8] O. Ileri, D. Samardzija, T. Sizer, N. B. Mandayam, Demand responsive pricing and competitive spectrum allocation via a spectrum server, in: Proceedings of IEEE International Symposium on New Frontiers in Dynamic Spectrum Access Networks (DySPAN), 2005, pp. 194-202.

[9] M. Pan, S. Liang, H. Xiong, J. Chen, G. Liu, A novel bargaining based dynamic spectrum management scheme in reconfigurable systems, in: Proceedings of IEEE ICSNC, 2006, p. 54. 
[10] S. Gandhi, C. Buragohain, L. Cao, H. Zheng, S. Suri, A general framework for wireless spectrum auctions, in: Proceedings of IEEE DySPAN, 2007, pp. 22-33.

[11] D. Niyato, E. Hossain, Competitive spectrum sharing in cognitive radio networks: a dynamic game approach, IEEE Transactions on Wireless Communications 7 (7) (2008) 2651-2660.

[12] D. Niyato, E. Hossain, Competitive pricing for spectrum sharing in cognitive radio networks: dynamic game, inefficiency of Nash equilibrium, and collusion, IEEE Journal on Selected Areas in Communications 26 (1) (2008) 192-202.

[13] D. Niyato, E. Hossain, Market-equilibrium, competitive, and cooperative pricing for spectrum sharing in cognitive radio networks: analysis and comparison, IEEE Transactions on Wireless Communications 7 (11) (2008) $4723-4283$.

[14] D. Niyato, E. Hossain, A microeconomic model for hierarchical bandwidth sharing in dynamic spectrum access networks, IEEE Transactions on Computers 59 (7) (2010) 865-877.

[15] M. Hassan, G. Karmakar, J. Kamruzzaman, Reputation and user requirement based price modeling for dynamic spectrum access, IEEE Transactions on Mobile Computing 13 (9) (2014) 2128-2140.

[16] Credit rating agency, http://en.wikipedia.org/wiki/Credit_rating_ agency, [Online; accessed 15-October-2014] (2014).

[17] D. Niyato, E. Hossain, Spectrum trading in cognitive radio networks: a market-equilibrium-based approach, IEEE Wireless Communications 15 (6) (2008) 71-80.

[18] O. Simeone, I. Stanojev, S. Savazzi, Y. Bar-Ness, U. Spagnolini, R. Pickholtz, Spectrum leasing to cooperating secondary ad hoc networks, IEEE Journal on Selected Areas in Communications 26 (1) (2008) 203-213. 
[19] D. Xu, X. Liu, Z. Han, A two-tier market for decentralized dynamic spectrum access in cognitive radio networks, in: Proceedings of IEEE SECON, 2010, pp. 1-9. 
[28] D. Joanes, Reject inference applied to logistic regression for credit scoring, IMA Journal of Management Mathematics 5 (1) (1993) 35-43.

[29] K. Leonard, Empirical Bayes analysis of the commercial loan evaluation process, Statistics \& probability letters 18 (4) (1993) 289-296.

[30] E. Laitinen, Predicting a corporate credit analyst's risk estimate by logistic and linear models, International Review of Financial Analysis 8 (2) (1999) $97-121$.

[31] S. Westgaard, N. Van der Wijst, Default probabilities in a corporate bank portfolio: A logistic model approach, European journal of operational research 135 (2) (2001) 338-349.

[32] T. Lee, I. Chen, A two-stage hybrid credit scoring model using artificial neural networks and multivariate adaptive regression splines, Expert Systems with Applications 28 (4) (2005) 743-752.

[33] D. Hosmer, S. Lemeshow, Applied logistic regression, Vol. 354, WileyInterscience, 2000.

[34] J. Liu, V. Issarny, Enhanced reputation mechanism for mobile ad hoc networks, in: Trust management, Springer, 2004, pp. 48-62.

[35] M. Hassan, G. Karmakar, J. Kamruzzaman, Dynamic spectrum trading model for cognitive radio networks, Ph.D. thesis, Faculty of Information Technology, Monash University, Australia. (2013).

[36] L. Mui, M. Mohtashemi, A. Halberstadt, A computational model of trust and reputation, in: Proceedings of IEEE Hawaii International Conference on System Sciences (HICSS), 2002, pp. 2431-2439.

[37] S. Kalepu, S. Krishnaswamy, S. Loke, Reputation= f (user ranking, compliance, verity), in: Proceedings of IEEE International Conference on Web Services, 2004, pp. 200-207. 
[38] W. Sherchan, S. Loke, S. Krishnaswamy, Explanation-aware service selection: rationale and reputation, Service Oriented Computing and Applications 2 (4) (2008) 203-218.

[39] S. Landon, C. E. Smith, Quality expectations, reputation, and price, Southern Economic Journal 64 (3) (1998) 628-647.

[40] E. W. Anderson, M. W. Sullivan, The antecedents and consequences of customer satisfaction for firms, Marketing science 12 (2) (1993) 125-143.

[41] T. W. Andreassen, B. Lindestad, The effect of corporate image in the formation of customer loyalty, Journal of Service Research 1 (1) (1998) 82-92.

[42] J. M. Spiteri, P. A. Dion, Customer value, overall satisfaction, end-user loyalty, and market performance in detail intensive industries, Industrial Marketing Management 33 (8) (2004) 675-687.

[43] PayPal seller protection policy, https://www.paypal.com/webapps/mpp/ security/seller-protection-faq, [Online; accessed 08-January-2014] (2014).

[44] Blacklisted buyers, http://badbuyerlist.org/, [Online; accessed 08January-2014] (2014).

[45] Chargebacks, https://www.paypal.com/au/webapps/mpp/security/ sell-chargebackfaq, [Online; accessed 08-January-2014] (2014).

[46] P. Dwyer, Measuring the value of electronic word of mouth and its impact in consumer communities, Journal of Interactive marketing 21 (2) (2007) $63-79$.

[47] M. Weiss, P. Krishnamurthy, L. E. Doyle, K. Pelechrinis, When is electromagnetic spectrum fungible?, in: Proceedings of IEEE International Symposium on Dynamic Spectrum Access Networks (DYSPAN), 2012, pp. 
[48] H. Varian, Microeconomic analysis, Norton New York, 1992.

[49] R. J. Lavery, Throughput optimization for wireless data transmission, Master's thesis, Polytechnic University (June 2001).

[50] C. Caicedo, M. Weiss, The viability of spectrum trading markets, Communications Magazine, IEEE 49 (3) (2011) 46-52. doi:10.1109/MCOM.2011. 5723799 .

[51] Mobile network operators of India, http://en.wikipedia.org/wiki/ Mobile_network_operators_of_India, [Online; accessed 16-October2014] (2014).

835 [52] P. Glendinning, Stability, instability and chaos: an introduction to the theory of nonlinear differential equations, Cambridge University Press, 1994.

[53] J. C. Sprott, Chaos and time-series analysis, Oxford University Press, 2003. 